\title{
Publicações de teatro em 2017
}
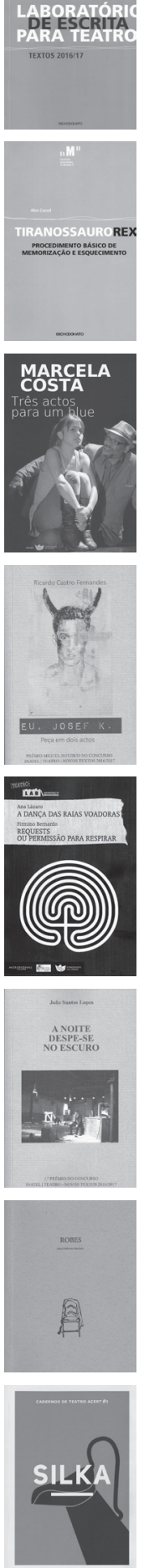

\author{
LISTA COMPILADA POR SEBASTIANA FADDA
}

\section{PEÇAS ORIGINAIS (OU VOLUMES DE PEÇAS) EM PRIMEIRA EDIÇÃO}

AA. VV., Laboratório de Escrita para Teatro: Textos 2016/17 (Mãos ao Alto: Ensaios para entrar no paraíso, de Cecília Ferreira; Os Lugares de Onde Vemos Sentados, de Fernando Giestas; Os Ratos, de Isabel Milhanas Machado; Treva ou Os Princípios da Higiene Funcional, de Sabrina D. Marques), coord. Rui Pina Coelho, Lisboa, Teatro Nacional D. Maria II / Bicho do Mato, 2017.

ANDRÉ, Raquel, Colecção de Amantes. Vol. I, ed. bilingue português/inglês, trad. Joana Frazão, Teatro Nacional D. Maria II / Bicho do Mato, 2017.

BAptista, Sónia, Querer do Corpo, Peso (e outros textos) [A Falha de onde a Luz / Assentar sobre a Subida das Águas], Évora, Não (Edições), Colecção Cénica n. o 2, 2017.

СА ВАÇ A, Ricardo, Stormi-Quiroga, Évora, Editora Licorne, 2017.

- Depois da Última Página / Náufragos, Vila Nova de Famalicão, Adab Edições, 2017.

CAssal, Alex, Tiranossauro Rex: Procedimento básico de memorização e esquecimento, Lisboa, Teatro Nacional D. Maria II / Bicho do Mato, 2017.

Correia, Romeu, Comédia de Maus Costumes, Casal de Cambra, Oro [Caleidoscópio], 2017.

Correia, Tiago, Pela Água, Grande Prémio de Teatro Português SPA / Teatro Aberto 2016, Lisboa, Sociedade Portuguesa de Autores / Imprensa Nacional-Casa da Moeda, 2017.

Cost A, Marcela, Três Actos para Um Blue (Stormy Weather, Summertime e September Song / A Canção de Setembro), Lajes do Pico, Companhia das Ilhas, AzulCobalto, n. ${ }^{0}$ 19, 2017.

FERnAndes, Ricardo Castro, Eu, Josef $K$. (Prémio Miguel Rovisco Fundação INATEL | Teatro Novos Textos 2016/2017), Lisboa, Fundação INATEL, 2017.

GARDEAZABAL, José, Trilogia do Olhar: Televisão / Regras para Fotografar Animais / Cinema Mudo, Lisboa, Sociedade Portuguesa de Autores / Imprensa Nacional-Casa da Moeda, 2017.

Godinho, Sérgio, Eu Tu Ele Nós Vós Eles! / O Corvo e a Raposa, Lisboa, Sociedade Portuguesa de Autores / Imprensa Nacional-Casa da Moeda, 2017.

LÁzA Ro, Ana / BERnARdo, Firmino, A Dança das Raias Voadoras [Ana Lázaro] / Requests ou Permissão para Respirar [Firmino Bernardo], Lajes do Pico, Companhia das Ilhas / Teatro Meridional / Centro de Estudos de Teatro - LAB Laboratório de Dramaturgia, AzulCobalto, n. ${ }^{\circ} 18,2017$.

LOPES, João Santos, A Noite Despe-se no Escuro (Grande Prémio Fundação INATEL | Teatro - Novos Textos 2016/2017), Lisboa, Fundação INATEL, 2017.

MACHADO, Isabel Milhanas, Robes, Lisboa, Casa-Mãe, 2017.

MARTins, José Rui, Silka, [a partir do conto de Ilse Losa], pref. de João Maria André, texto de José Rui Martins e Carlos Silva, Tondela, Acert, Cadernos de Teatro, n. ${ }^{\circ} 1,2017$.

MARTINs, José Rui / José, Pompeu, À Roda da Noite [guião original e adaptação livres a partir de contos de Mia Couto], pref. João Maria André, texto de José Rui Martins, Tondela, Acert, Cadernos de Teatro, n. ${ }^{\circ}$ 2, 2017.

MEND ONÇA. Guilherme, Um Pássaro É mais do Que a Sua Jaula [monólogo dramático], Lisboa, Edições Colibri, 2017.

NEgreiros, José de Almada, Teatro Escolhido (Antes de Começar / Deseja-se Mulher / O Público em Cena / Aquela Noite / Aqui Cáucaso), ed. Fernando Cabral Martins e Luís Manuel Gaspar, introdução de Fernando Cabral Martins, Lisboa, Assírio \& Alvim, Almada Breve, 2017. 

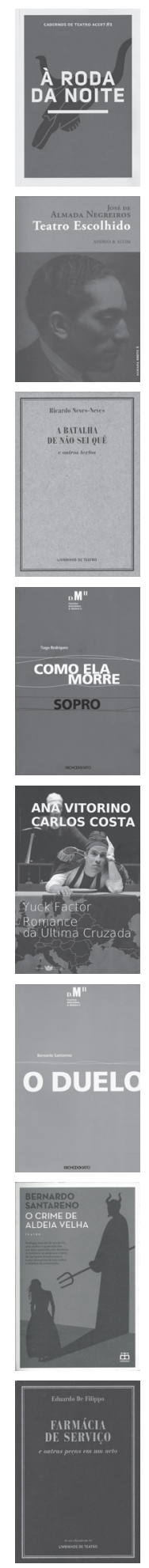

NEVES-NEVEs, Ricardo, A Batalha de Não-sei-quê e outros textos (Noé / Ilha flutuante / Entraria nesta sala...), Lisboa, Artistas Unidos / Livros Cotovia, Livrinhos de Teatro n. ${ }^{0}$ 110, 2017.

PIREs, Jacinto Lucas, Exactamente Antunes, Vila Nova de Famalicão / Porto, Húmus / Teatro Nacional São João, Colecção Teatro Nacional São João, 2017.

RochA, Ana, A Origem do Mundo, Vila Nova de Famalicão, Adab Edições, 2017.

Rodrigues, Tiago, Como Ela Morre / Sopro, Lisboa, Teatro Nacional D. Maria II / Bicho do Mato, 2017.

SILVA, Pedro Augusto de Sousa e, Gomes Freire: Peça histórica em 4 actos e 6 quadros, coord. Eugénia Vasques e Mário J. Freire da Silva, Amadora, Direção de História e Cultura Militar e da Escola Superior de Teatro e Cinema/IPL, 2017.

vitorino, Ana / costA, Carlos, Yuck Factor / Romance da Última Cruzada, AzulCobalto, n. ${ }^{\circ}$ 20, 2017.

\section{PEÇAS ORIGINAIS EM REEDIÇÃO}

MACHADO, Carlos Alberto, Teatro Reunido: 200o-2010 (Os Nomes que Faltam / Ficava tão bem Naquele Canto da Sala / A Felicidade Ideal / Transportes e Mudanças / Avesso / Restos. Interiores / Aquitanta / Hamlet \& Ofélia / Ponho Palavras na Minha Cabeça / s Cervejas para o Virgílio / Os Bravos do Kosovo / Hoje não Há Música / O Sentido da Vida), pref. Rui Pina Coelho, Lajes do Pico, Companhia das Ilhas, AzulCobalto, n.o 9, [2014] 2017. [reimpressão]

Monteiro, Luísa / romão, Valério / coelho, Rui Pina, Irina / Macha / Olga / Olya, (Luísa Monteiro: Irina, Valério Romão: Macha, Rui Pina Coelho: Olga), Lisboa, Não (Edições), Colecção Cénica, n. ${ }^{0}$ 1, [2016] 2017. [reimpressão]

SAntAREno, Bernardo, O Duelo, Lisboa, Teatro Nacional D. Maria II / Bicho do Mato, [1. a ed.: Lisboa, Ática, 1961] 2017.

-, O Crime da Aldeia Velha, Silveira, E-primatur, [1. ${ }^{\mathrm{a}}$ ed.: Lisboa, Ática, 1961] 2017.

\section{TRADUÇÕES}

BAKER, Annie, O Cinema, trad. e rev. Francisco Frazão, Lisboa, Artistas Unidos / Livros Cotovia, Livrinhos de Teatro n. ${ }^{\circ}$ 104, 2017.

CASonA, Alejandro, Coroa de Amor e Morte, trad. Maria João da Rocha Afonso, Casal de Cambra, Oro [Caleidoscópio], 2017.

DE FILIPPO, Eduardo, Farmácia de Serviço e outras peças em um acto (Sik Sik, o Artífice mágico / Filosoficamente / Esses Figurões de Há zo Aos / Gennariello / Adeus, Quinto Andar! / Perigosamente / Amizade / Dor Fechada a Sete Chaves), trad. José Colaço Barreiros, Lisboa, Artistas Unidos / Livros Cotovia, Livrinhos de Teatro n. ${ }^{0}$ 103, Os Clássicos, 2016.

DEL FEDERICO, Matias, Em terapia / Somos childfree, trad. Ângelo Ferreira de Sousa, Lisboa, Artistas Unidos / Livros Cotovia, Livrinhos de Teatro n. ${ }^{\circ}$ 113, 2017.

DÜRRENMAT T, Friedrich, A Visita da Velha Senhora, trad. João Barrento, Lisboa, Artistas Unidos / Livros Cotovia, Livrinhos de Teatro n. ${ }^{\circ}$ 106, 2017.

FossE, Jon, Calor e outras peças (Ylajali / A Rapariga da Gabardina Amarela / Viver Secretamente), trad. Pedro Porto Fernandes, Lisboa, Artistas Unidos / Livros Cotovia, Livrinhos de Teatro n. ${ }^{\circ} 109,2017$.

кнатів, Mohamed El, Acabar em Beleza, trad. Alexandra Moreira da Silva, Lisboa, Teatro Nacional D. Maria II / Bicho do Mato, 2017.

LORCA, Federico Garcia, Mariana Pineda, trad. Miguel Martins, Évora, Não (Edições), Colecção Cénica n. ${ }^{\circ} 3,2017$. 

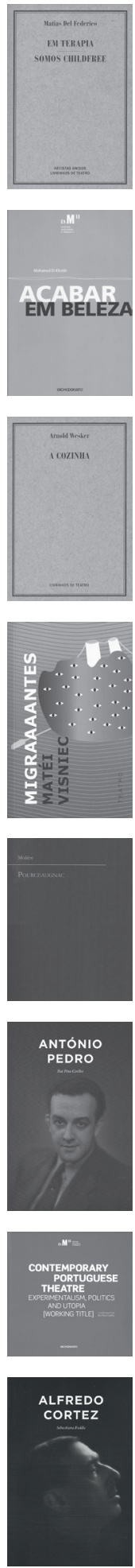

MIRó, Pau, Vitória, trad. Joana Frazão, Lisboa, Artistas Unidos / Livros Cotovia, Livrinhos de Teatro n. ${ }^{\mathrm{o}} 108,2017$.

MOLiÈre, Pourceaugnac, versão portuguesa do século XVIII, ed. José Camões, Marie-Noelle Ciccia e Ariadne Nunes, Lisboa, Centro de Estudos de Teatro, 2017.

Mülle R, Heiner, A Missão / Mauser, trad. Maria Adélia Silva Melo e Jorge Silva Melo, Lisboa, Artistas Unidos / Livros Cotovia, Livrinhos de Teatro n. ${ }^{\circ}$ 105, 2017.

tolcachir, Claudio, A Omissão da Família Coleman / Terceiro Corpo, trad. Rita Bueno Maia e Maria João Machado, Lisboa, Artistas Unidos / Livros Cotovia, Livrinhos de Teatro n. ${ }^{\circ} 111$, 2017.

VERonese, Daniel, Mulheres Sonharam Cavalos / De Carro, trad. Ângelo Ferreira de Sousa, Lisboa, Artistas Unidos / Livros Cotovia, Livrinhos de Teatro n. ${ }^{\circ}$ 112, 2017.

visbiec, Matéi, Migraaaantes, trad. Ângela Pardelha, Almada, Companhia de Teatro de Almada / Livros de Areia, 2017.

Wesker, Arnold, A Cozinha, trad. Maria João da Rocha Afonso, Lisboa, Artistas Unidos / Livros Cotovia, Livrinhos de Teatro n. ${ }^{\circ}$ 107, 2017.

\section{TRADUÇÕES EM REEDICุÃO}

BRECHT, Bertolt, Teatro 2 (A Vida de Eduardo II de Inglaterra / Um Homem é um Homem / Ascenção e Queda da Cidade de Mahagonny / A Ópera de Três Vinténs), introdução de Vera San Payo de Lemos, trad. Manuel Resende ( $A$ Vida de Eduardo II de Inglaterra), António Conde (Um Homem é um Homem), João Lourenço e Vera San Payo de Lemos (Ascenção e Queda da Cidade de Mahagonny), João Lourenço e Vera San Payo de Lemos com a colaboração de José Fanha (A Ópera de Três Vinténs), Lisboa, Livros Cotovia, [1. ${ }^{\mathrm{a}}$ ed.: 2004] 2006 [2. ${ }^{\mathrm{a}}$.

ShAKespeARE, William, A Tempestade seguido de O Mar e o Espelho, de W. H. Auden, trad. Daniel Jonas, Lisboa, Edições Cotovia, [1. ${ }^{\mathrm{a}}$ ed.: 2009] 2017 [3. $\left.{ }^{\mathrm{a}}\right]$.

\section{ESTUDOS / DOCUMENTOS}

ANDRÉ, João Maria, Jogo, Corpo e Teatro: A Arte de Fazer Amor com o Tempo, Coimbra, Edições Angelus Novus, 2017.

CALAdo, Alexandre Pieroni (coord.), Os Pontos no Teatro Nacional D. Maria II, com depoimentos e textos de Cristina Vidal e João Coelho, Helena Diogo, Joaquim Samora, André Teodósio, Carlos Avilez, Carmen Dolores, João Lourenço, Jorge Silva Melo, José Raposo, Miguel Loureiro, Eugénia Vasques e Alexandre Pieroni Calado, Lisboa, Teatro Nacional D. Maria II / Bicho do Mato, 2017.

coelho, Rui Pina, António Pedro, Lisboa, Centro de Estudos de Teatro-Faculdade de Letras da Universidade de Lisboa / Teatro Nacional D. Maria II / Teatro Nacional São João / Imprensa Nacional-Casa da Moeda, Colecção Biografias do Teatro Português [coord. científica M. J. Brilhante e A. I. Vasconcelos], n. ${ }^{\circ}$ 4, 2017.

- (coord.), Teatro Português Contemporâneo: Experimentalismo, política e utopia [Título Provisório], (textos de Rui Pina Coelho, Gustavo Vicente, Jorge Louraço Figueira, Ana Pais, Maria Sequeira Mendes, Rita Martins, Eunice Tudela de Azevedo, Paula Gomes Magalhães, Ana Bigotte Vieira), Lisboa, Teatro Nacional D. Maria II / Bicho do Mato, 2017.

- (coord.), Contemporary Portuguese Theatre: Experimentalism, politics and utopia [Working Title], (texts by Rui Pina Coelho, Gustavo Vicente, Jorge Louraço Figueira, Ana Pais, Maria Sequeira Mendes, Rita Martins, Eunice Tudela de Azevedo, Paula Gomes Magalhães, Ana Bigotte Vieira), trad. Rosa Churcher Clarke, Scott Culp, Lisboa, Teatro Nacional D. Maria II / Bicho do Mato, 2017. 

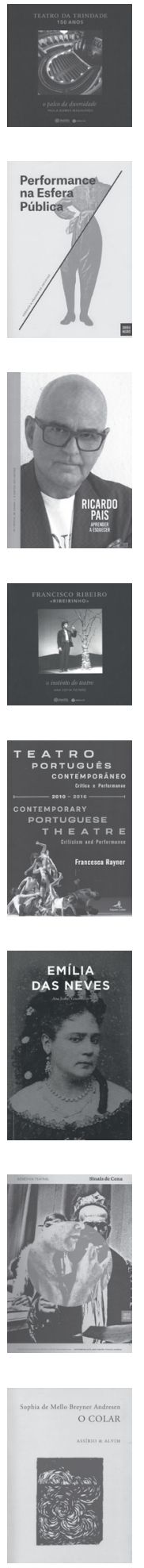

Costa, Ana Paula, Nome: Natália, Casal de Cambra, Oro [Caleidoscópio], 2017.

FADDA, Sebastiana, Alfredo Cortez, Lisboa, Centro de Estudos de Teatro - Faculdade de Letras da Universidade de Lisboa / Teatro Nacional D. Maria II / Teatro Nacional São João / Imprensa Nacional-Casa da Moeda, Colecção Biografias do Teatro Português [coord. científica M. J. Brilhante e A. I. Vasconcelos], n. ${ }^{2}$ 2, 2017.

MAgAlhães, Paula Gomes, Teatro da Trindade 150 Anos: O palco da diversidade, Lisboa, INATEL / Editora Guerra \& Paz, 2017.

Moreira, Joana Miguel, O Teatro no Porto no Período entre Guerra: Os Teatros Carlos Alberto e São João (1914-1945), Caleidoscópio / Centro República, 2017.

OliveIRA, Fernando Matos / ARRAIs, Thiago (ed.), Ensaios Ruminantes. Sobre a obra performativa de Patrícia Portela, com textos dos editores e de Ana Pais, Cláudia Galhós, Sónia Baptista, Mickael de Oliveira, Isabel Garcez, Mónica Guerreiro, Maria João Faustino, Carlos Emílio Corrêa Lima, entre outros, Coimbra, Imprensa da Universidade de Coimbra, Coleção Olhares, 2017.

PAIs, Ana (org.), Performance na Esfera Pública, com textos e colaborações de Bojana Cvejić e Ana Vujanović, Carla Cruz, Sandra Guerreiro Dias, David Helbich, Isabel Nogueira, Claire Bishop, Eleonora Fabião, Sevi Bayaktar, Maria Andueza Olmedo, Christof Migone, Rui Mourão, Liliana Coutinho \& Catherine Wood, Peggy Phelan, Ana Pais, Ana Bigotte Vieira, Leif Elggren/KREV, Ana Borralho \& João Galante, Sílvia Pinto Coelho, João Macdonald, Christine Greiner, Andrea Maciel, Paulo Raposo e Guillermo Gómez-Peña, Lisboa, Orfeu Negro, 2017.

PAIs, Ricardo, Aprender a Esquecer, [33. ${ }^{\circ}$ Festival de Almada: Ciclo O Sentido dos Mestre] Almada, Companhia de Teatro de Almada, 2017.

PATrÂo, Ana Sofia, Francisco Ribeiro. «Ribeirinho»: O instinto do teatro, Lisboa, INATEL / Editora Guerra \& Paz, 2017.

PEREIRA, Silvina, Dramas Imperfeitos. Teatro Clássico Português: um repertório a descobrir, pref. José Augusto Cardoso Bernardes, Lisboa, Eos Edições / Centro de Estudos Clássicos da Faculdade de Letras da Universidade de Lisboa, 2017.

RAYNER, Francesca, Teatro Português Contemporâneo: Crítica e Performance / Contemporary Portuguese Theatre: Criticism and Performance.2006-2010, Lisboa, Edições Colibri, 2017.

RODRIgues, Paulo Renato (coord.), Ildefonso Valério: Uma vida com teatro dentro, Vila Franca de Xira, Câmara Municipal de Vila Franca de Xira, 2017.

vasconcelos, Ana Isabel, Emília das Neves, Lisboa, Centro de Estudos de Teatro - Faculdade de Letras da Universidade de Lisboa / Teatro Nacional D. Maria II / Teatro Nacional São João / Imprensa Nacional-Casa da Moeda, Colecção Biografias do Teatro Português [coord. científica M. J. Brilhante e A. I. Vasconcelos], n. ${ }^{\circ} 4,2017$.

\section{ADENDA À LISTA PUBLICADA NA SINAIS DE CENA, SÉRIE II, N.² 2 (2017)}

ANDRESEN, Sophia de Mello Breyner, O Colar, Porto, Porto Editora [1. ${ }^{\mathrm{a}}$ ed.: Lisboa, Editorial Caminho, 2001], [2012 1.a] 2016. [peça original em reedição]

СнамвеL, Filipa, Intermedialidade Cénica: As margens da experiência estética do teatro, Amadora, ESTC Edições, 2016, disponível em https://editora.estc.ipl.pt/. [ensaio]

Costa, Maria Manuel, No Poial ao Entardecer, Lisboa, Edições Vieira da Silva, 2016. [peça original]

DURAs, Marguerite, Dias Inteirosnas Árvores / Savannah Bay, trad. Vieira de Lima e António de Barahona, Lisboa, Artistas Unidos / Livros Cotovia, Livrinhos de Teatro n. ${ }^{0}$ 101, 2016. [tradução]

GARCIA, Rodrigo, Comprei uma Pá no Ikea para Cavar a Minha Sepultura e outras peças (Daisy / A floresta é nova e está cheia de vida), trad. Tiago Rodrigues e John Romão, Lisboa, Artistas Unidos / Livros Cotovia, Livrinhos de Teatro n. ${ }^{\circ}$ 102, 2016. [tradução]

KraUs, Karl, Os Últimos Dias da Humanidade, trad. António Sousa Ribeiro, Vila Nova de Famalicão / Porto, Húmus / Teatro Nacional São João, Colecção Teatro Nacional São João, 2016. [tradução] 
Neves, Abel, Ainda o Último Judeu e os Outros / Ossman, Vila Nova de Famalicão, Adab Edições, 2016. [peça original]

OLIVA, João Luís, ACERT XL. O Fio, a Trama e a Urdidura, pref. João Maria André, Porto, Edições Afrontamento, 2016. [estudo / documento]

RED ONDO Júnior, José Rodrigues, $O$ Atrevido, [comédia em 3 actos], ed. fac-similada da $1 .^{\mathrm{a}}$ ed. [Lisboa, Imp. Sociedade Nacional de Tip., 1945], pref. Jorge de Faria, Casal de Cambra, Oro [Caleidoscópio] / AIC [Associação Internacional de Cenografia], 2016. [peça original em reedição]

SAnTARE No, Bernardo, O Inferno, Silveira, E-primatur, [1. a ed: Lisboa, Ática, 1967], 2016. [peça original em reedição]

SHAKESPEARE, William, Muito Barulho por Nada, trad. Sophia de Mello Breyner Andresen, pref. Luis Miguel Cintra, introdução João Almeida Flor, Lisboa, Assírio \& Alvim, 2016. [tradução]

Sinais de Cena, série II, n. ${ }^{\circ} 2$ (2017), dir. Rui Pina Coelho, Lisboa, Centro de Estudos de Teatro em colaboração com a Associação Portuguesa de Críticos de Teatro, Lisboa, Orfeu Negro.

VEnTURA, Ruy, Detergente (poema), Évora, Editora Licorne, 2016. [peça original]

VICENTE, Gil, Auto de Moralidade da Embarcação do Inferno, in Programa do espectáculo Embarcação do Inferno, de Gil Vicente, produção Escola da Noite / Cendrev, Coimbra/Évora, s/d [2016]. [Peça original em reedição]

\section{ERR ATA}

Os títulos que seguem foram anunciados na Sinais de Cena, série II, n. ${ }^{\circ}$ (2017), mas ainda não chegaram a ser editados:

FARIA, Cristina (coord.), O Nacional Está a Arder! O incêndio de 1964 e o fim de uma época, textos de António Morgado, Cristina Faria, Isabel Vidal, Luís Soares Carneiro, Lisboa, Teatro Nacional D. Maria II / Imprensa Nacional-Casa da Moeda, 2016.

VARGAS, Carlos / MASCARENHAS-MATEUs, João (eds.), TNDM II - Arquitetura e Património, textos de José Augusto-França, José Monterroso Teixeira, Milton Pacheco, Erico da Costa, Rui Dâmaso, Pedro Costa, Sérgio Henriques, Manuel Alexandre, Paulo Prata Ramos, Pedro Fidalgo e Carlos Vargas, Lisboa, Teatro Nacional D. Maria II / Imprensa Nacional-Casa da Moeda, 2016.

\section{ADENDA À LISTA PUBLICADA NA SINAIS DE CENA N. 1 (2016)}

Lorca, Federico García, Assim Que Passarem Cinco Anos, trad. Cláudio Castro Filho, Coimbra, Imprensa da Universidade de Coimbra, Colecção Dramaturgia, 2014. [tradução]

\section{ADENDA À LISTA PUBLICADA NA SINAIS DE CENA N. 21 (2014)}

Andresen, Sophia de Mello Breyner, O Colar, prefácio de Luis Miguel Cintra, Lisboa, Assírio \& Alvim [1. ${ }^{\mathrm{a}}$ ed. Lisboa, Editorial Caminho, 2001], 2013. [peça original em reedição, com prefácio original]

\section{ADENDA À LISTA PUBLICADA NA SINAIS DE CENA N. 19 (2013)}

DJE RAss I, Carl, Cálculo: Peça em dois actos, Coimbra, Imprensa da Universidade de Coimbra, Colecção Dramaturgia, 2012. 
Ros A, Armando Nascimento, Três Peças Mitocríticas I: Um Édipo-O drama ocultado. Mitodrama Fantasmático em Um Acto, Coimbra, Imprensa da Universidade de Coimbra, Colecção Dramaturgia, 2012. [peça original]

Ros A, Armando Nascimento, Três Peças Mitocríticas III: O eunuco de Inês de Castro. Teatro no País dos Mortos, Coimbra, Imprensa da Universidade de Coimbra, Colecção Dramaturgia, 2012. [peça original]

TORRADO, António, Atirem-se ao Ar! O que ninguém contou de uma viagem histórica, Lisboa, Editorial Caminho, 2012. [peça original]

\section{ADENDA À LISTA PUBLICADA NA SINAIS DE CENA N. 17 (2012)}

TORRADO, António, O Homem sem Sombra, Lisboa, Editorial Caminho [1. ${ }^{\mathrm{a}}$ ed.: 2005], 2011. [peça original em reedição]

ADENDA À LISTA PUBLICADA NA SINAIS DE CENA N. 15 (2011)

CARDoso, João Paulo Seara, Cinderela, Porto, Porto Editora, 2010. [peça original] 